\title{
Oblique powder blasting for three-dimensional micromachining of brittle materials
}

\author{
E. Belloy*, A. Sayah, M.A.M. Gijs \\ Institute of Microsystems, Swiss Federal Institute of Technology Lausanne, CH-1015 Lausanne EPFL, Switzerland
}

\begin{abstract}
We present oblique powder blasting as a three-dimensional micromachining technology for brittle materials. Powder blasting is a microfabrication process, based on the use of a pressurised air beam containing eroding $\mathrm{Al}_{2} \mathrm{O}_{3}$ particles. By varying the angle of incidence of the incoming particles to a substrate, covered by a mask, one can exploit the oblique slopes of micropatterned holes and mask underetching phenomena to generate new options for three-dimensional microstructuring. We have identified and quantified the role of secondary rebounding particles in mask underetching. (C) 2001 Elsevier Science B.V. All rights reserved.
\end{abstract}

Keywords: Sand blasting; Powder blasting; Microfabrication; Erosion; Etching

\section{Introduction}

Powder blasting is a very interesting technology for the realisation of three-dimensional microstructures in brittle materials like glass or silicon. This etching process is based on the eroding properties of $30 \mu \mathrm{m}$ size $\mathrm{Al}_{2} \mathrm{O}_{3}$ sharp particles projected at high speed against a target substrate. The origin of this high speed etching (typical etching rates of $100-1000 \mu \mathrm{m} / \mathrm{min}$ ) is the mechanical removal of matter by crack generation on the target surface [1,2]. Powder blasting using patterned protective masks has been developed for the patterning of microholes in large area glass substrates for flat display applications [1]. Recently, we have introduced this technique for the microfabrication of small-size microsystems, like cantilever accelerometer beams [3,4]. Due to the intrinsic simplicity and economic feasibility of the technique, it has a large application potential, even for high resolution $(<10 \mu \mathrm{m})$ patterning [5]. Powder blasting is mostly practised using a powder beam at normal incidence angle on the substrate $\left(\theta=90^{\circ}\right)$.

In this paper, we investigate in detail the basics of the oblique powder blasting process, by varying the angle of incidence of the powder beam to floatglass substrates $\left(\theta=80-40^{\circ}\right)$. We analyse the wall slopes of patterned holes and study mask underetching phenomena. Using a mathematical procedure, we are able to separate the erosion effects of primary impacting particles from secondary rebounding ones. We find that mask underetching is exclusively due to

\footnotetext{
* Corresponding author. Tel.: +41-21-693-6759; fax: +41-21-693-6670. E-mail address: eric.belloy@epfl.ch (E. Belloy).
}

secondary impacting particles and can be described by a simple trigonometric empirical relation. We think that the fundamental know-how on this oblique powder blasting process will create new options for three-dimensional microfabrication of brittle materials.

\section{Experimental set-up}

The erosion experiments were performed using a Texas Airsonics abrasive jet machine type HP-2, which is connected to a 6 bar pressurised air source. In this apparatus, the powder is dosed by a vibration feeder to the compressed air flux and transported by it to a nozzle through a rubber tube. As already introduced, the powder we used consists of $30 \mu \mathrm{m}$ size alumina sharp particles $\left(\mathrm{Al}_{2} \mathrm{O}_{3}\right)$.

For the erosion experiments, we put a protective metal mask with a thickness of $0.5 \mathrm{~mm}$, containing $3 \mathrm{~mm}$ wide square hole structures, onto a glass wafer. The mask is fixed on the surface of the substrate using an Apiezon ${ }^{\mathbb{R}}$ wax seal, to avoid mask lifting by the air jet. A schematic view of our set-up is shown in Fig. 1. It permits to vary the distance $d$ from the exit nozzle to the substrate from 1 to $9 \mathrm{~cm}$ and the angle of incidence $\theta$ of the nozzle from 30 to $90^{\circ}$.

\section{Experimental results and discussion}

\subsection{Hole sidewall profiles for $\theta=90^{\circ}$ and $\theta=50^{\circ}$}

When using a mask of square holes, powder blasting at normal incidence $\left(\theta=90^{\circ}\right)$ gives rise to a characteristic hole 


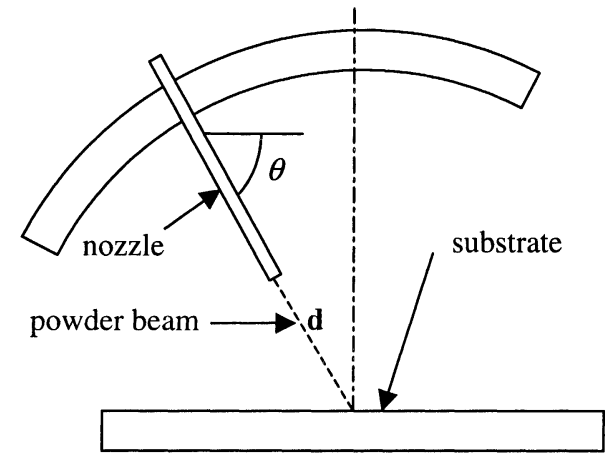

Fig. 1. Schematic view of the used powder blasting configuration. sidewall profile, which is a function of erosion time and hole depth. Fig. 2(a) presents a picture of a cross-section of a hole profile obtained after a $20 \mathrm{~s}$ long erosion experiment at normal incidence on a $1 \mathrm{~mm}$ thick glass substrate. The position of the mask during the etching process is schematically indicated. One can see that the resulting hole shape is symmetrical due to the uniform and normal exposure of the powder beam all over the hole structure. This specific hole shape is due to particle size effects, giving a reduced erosion close to the mask edge, in combination with the dependence of erosion efficiency on the particle impact angle to the surface, as explained in [6]. We will now show how the introduction of oblique powder blasting provides new possibilities for hole sidewall shape control. Fig. 2(b) shows a hole profile cross-section obtained by a uniform exposure of

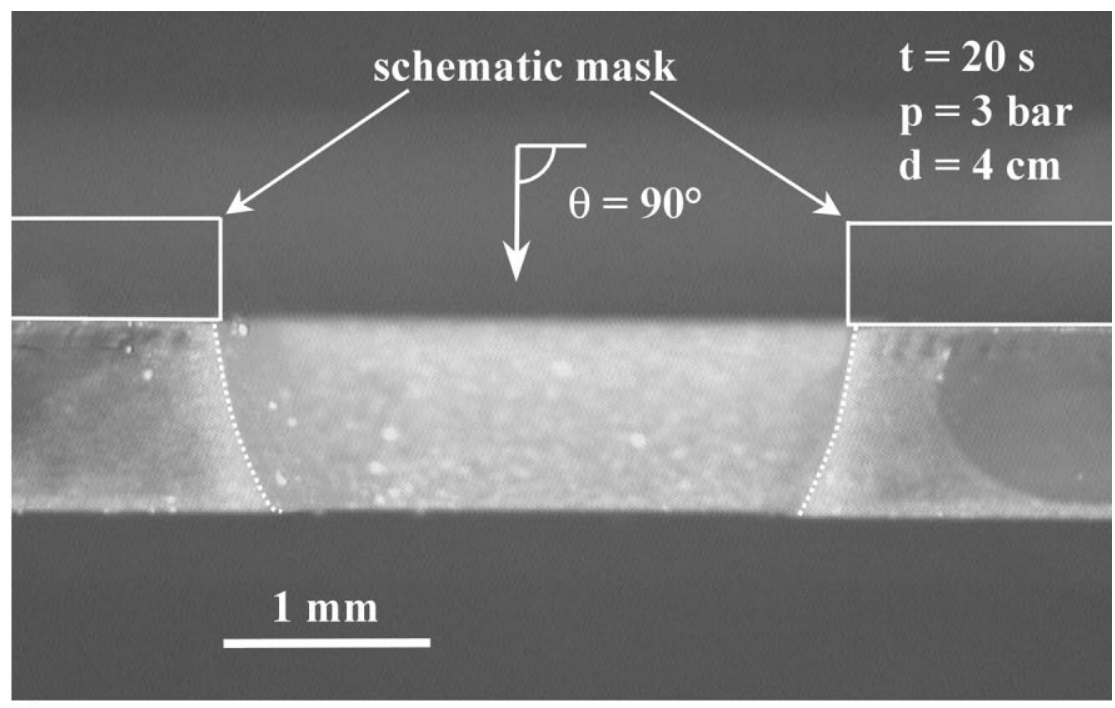

(a)

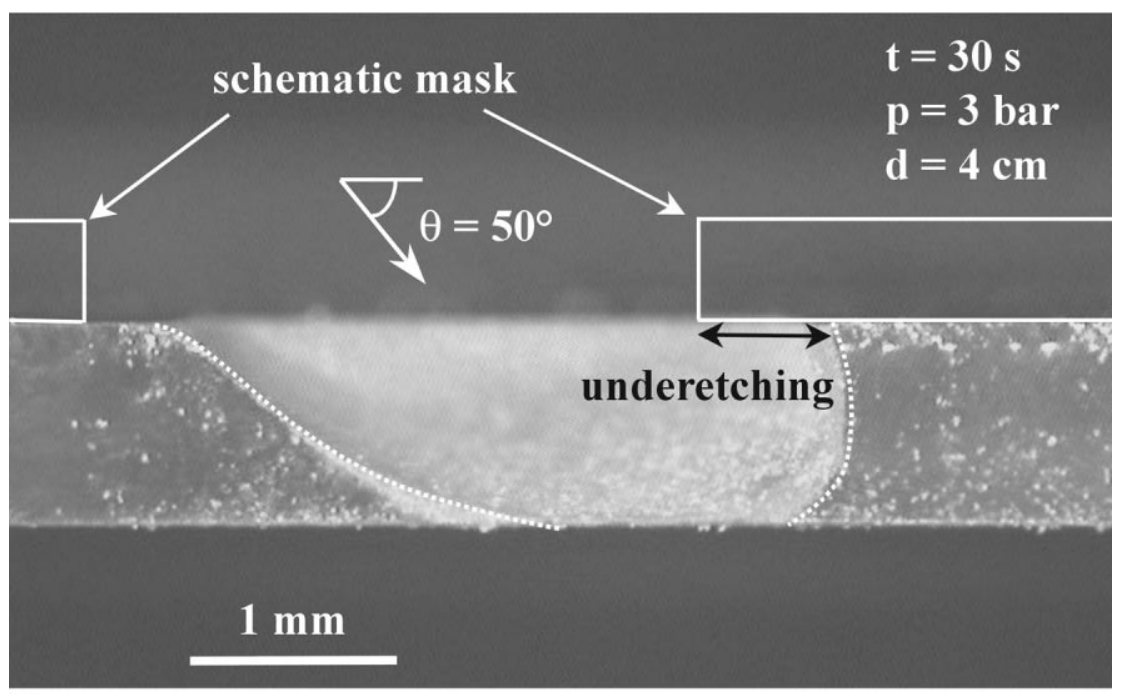

(b)

Fig. 2. (a) Profile picture of a normal powder blasted hole in glass. The position of the mask during erosion is schematically indicated. (b) Profile picture of an oblique powder blasted hole in glass using an eroding beam with an incidence angle $\theta=50^{\circ}$. The position of the mask during erosion is schematically indicated. 
the substrate to an oblique powder beam, with an angle $\theta=50^{\circ}$ (schematically indicated on the figure). Two main effects can be deduced from Fig. 2(b): the first one is a major difference between the sidewall angle on the left- and righthand side of the hole structure and the second one is the important mask underetching effect on the right. Mask underetching is defined as the distance the mask is underetched near the mask-substrate interface, as schematically indicated in the figure. While the wall profile on the left is the result of directly impacting (primary) particles from the powder beam, mask underetching necessarily is the result from secondary particles rebounding from the bottom of the hole structure. Erosion and wall slope resulting from primary particle impacts have already been studied for various angles of incidence and compared with a theoretical mathematical model [6]. In the next subsections, we study in depth the wall profile on the right side of the structure and identify there secondary particles as the major source of erosion.

\subsection{Underetching}

We have quantified the underetching effect for different etching times and incidence angles $\theta$ (see Fig. 3). One can see that the underetching effect is more important for the small impact angles. The curves increase continuously with the etching time and tend to saturate at a time scale corresponding to the etching time at which the substrate is completely etched through. After that time, the particles will not be able to rebound to the right sidewall, but will simply pass through the substrate, where they are evacuated. The observation of the underetching evolution of Fig. 3 is a first indication of the non-negligible contribution of secondary reflecting particles, as presented in Fig. 4.

The solid line profile of Fig. 4(a) represents a schematic hole shape, obtained after a normal powder blasting process resulting from primary particles only, as in Fig. 2(a). The dashed line represents the theoretical erosion profile (due to primary particles only), by introducing a non-normal angle of incidence to the substrate. It simply can be obtained by a correction of each point $(x ; y)$ of the normal profile as

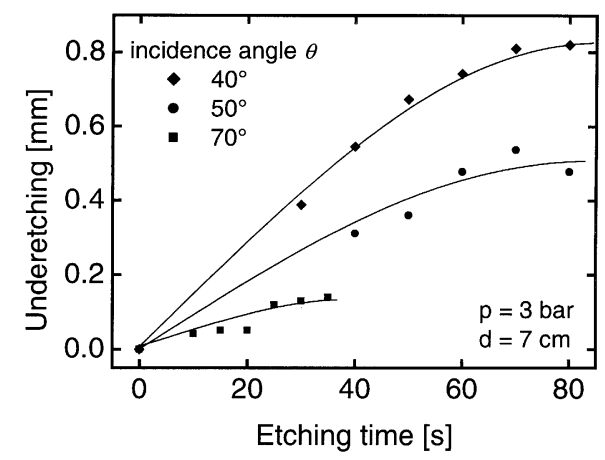

Fig. 3. Underetching as a function of the etching time for different angles of incidence $\theta$.

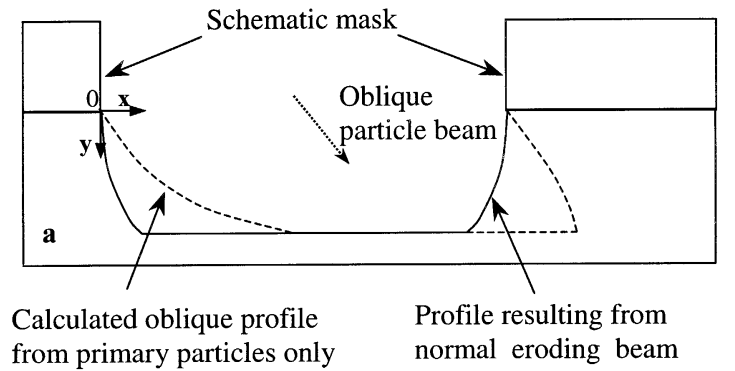

(a)

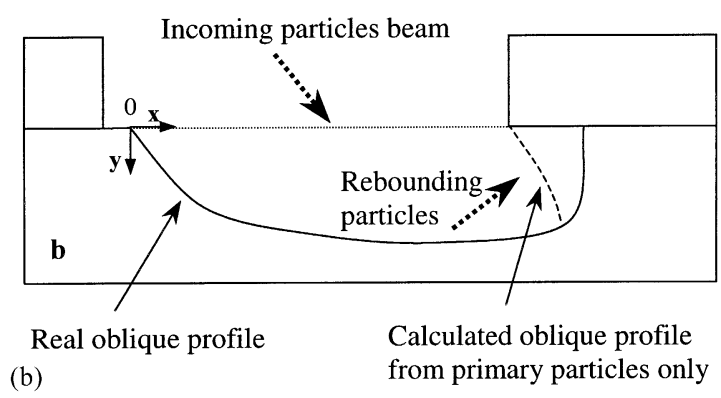

Fig. 4. (a) Schematic profile obtained with a normal particle beam (solid line) and a calculated profile for an oblique angle of incidence if only primary particles contribute to the erosion (dashed line). (b) Schematic experimental profile obtained with an oblique particle beam (solid line) and a calculated profile for an oblique angle of incidence if only primary particles contribute to the erosion (dashed line). One can see the underetching effect resulting from the impact of rebounding particles.

follows: $(x ; y)=>(x+y \tan (\theta) ; y)$, where $x$ - and $y$-axes are defined in Fig. 4. Fig. 4(b) shows a schematic view of an experimental profile of an oblique erosion process (solid line) and the theoretical profile (dashed line) of Fig. 4(a). The difference between the experimental profile and the theoretical one must be the result of erosion by secondary rebounding particles, as schematically indicated in Fig. 4(b). It is interesting to notice that only the right-hand side of the hole presents a difference between the experimental profile and the theoretical one. On the other hand, experimental and theoretical profiles are identical on the left side, where the erosion process results only from primary impacting particles.

\subsection{Quantification of the secondary particle erosion contribution}

Various sets of experimental hole profiles, obtained at different etching times, are presented by the solid lines in Fig. 5 for angles of incidence ranging from 40 to $80^{\circ}$. These profiles were obtained using a MikroCAD ${ }^{\circledR}$ optical profilometer system. ${ }^{1}$ In order to quantify the second particle erosion contribution on the right side of the hole, we need a reference profile obtained by the erosion of only primary

\footnotetext{
${ }^{1}$ Product information MikroCAD ${ }^{\circledR}$, GFMesstechnik GmbH, Berlin, Germany.
} 

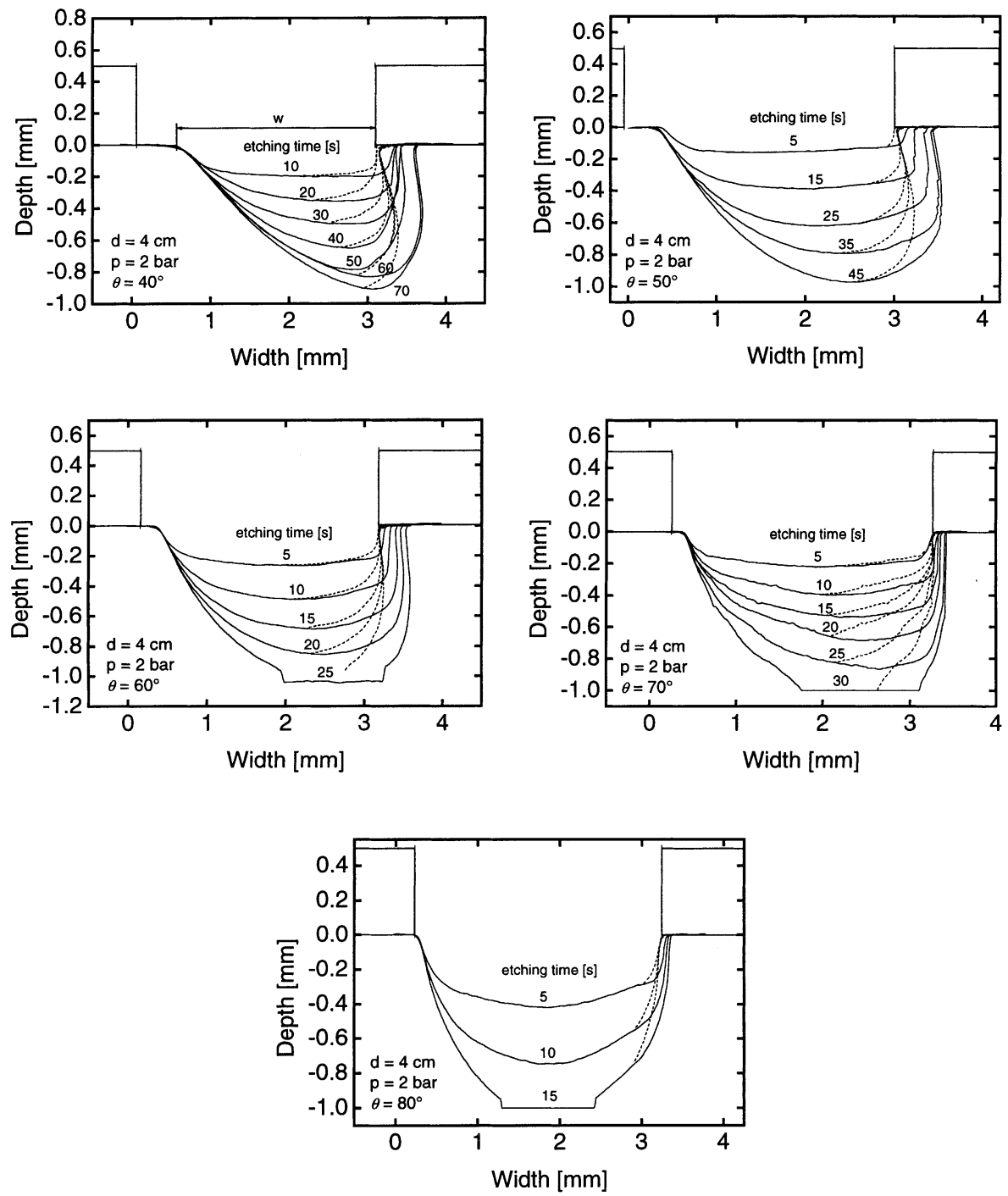

Fig. 5. Set of experimental profiles (solid lines) obtained after various etching times and for various angles of incidence of the powder beam. The dashed lines represent the corresponding theoretical profiles resulting from the erosion of primary impacting particles only.

impacting particles. As the left profile fulfils this requirement, we have systematically used it to reconstruct such reference. The dashed curves of Fig. 5 are obtained after three mathematical transformation steps of the left original shape, as follows:

1. The left oblique profile is transformed into a normal one by translating each point of the curve $(x ; y)$ : $(x ; y) \rightarrow(x-y \tan (\theta) ; y)=\left(x^{\prime} ; y\right)$, using the axis definition of Fig. 4(b). The resulting curve is a left erosion profile of primary particles at normal impact.

2. We then reconstruct the right-hand profile from the resulting curve of step (1). As the normal profile is symmetrical along the normal axis, we just create the mirror profile: $\left(x^{\prime} ; y\right)=>\left(-x^{\prime}+w ; y\right)=\left(x^{\prime \prime} ; y\right)$, with $w$ the width of the structured hole at the mask-substrate interface, as schematically indicated in Fig. 5.
3. We now need to reconstruct the right oblique profile from the resulting curve of step (2). This profile is the result of just primary particle impacts. It can be obtained by one more tranformation: $\left(x^{\prime \prime} ; y\right) \rightarrow\left(x^{\prime \prime}+y\right.$ $\tan (\theta) ; y)$.

The resulting curves, obtained after step (3), are shown in Fig. 5 as the dashed lines for each experimental profile. The strong difference between this theoretically calculated right oblique profiles and the experimental curves indicate the importance of erosion by secondary particle impacts. For a certain angle of incidence $\theta$, this difference increases with etching time; also the effect is larger for smaller $\theta$. The difference between the real experimental curves (solid lines) and the mathematically deduced ones (dashed lines) allows a precise quantification of the erosion contribution of the rebounding particles. 


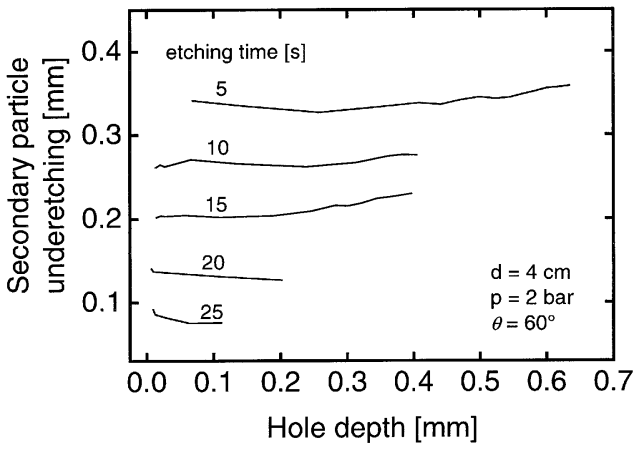

Fig. 6. Secondary particle erosion (horizontal difference between the solid and the dashed lines of Fig. 5) as a function of the hole depth for $\theta=60^{\circ}$ and at various etching times.

In order to quantify the contribution of secondary particles (see Fig. 4(b)), we have used the data of Fig. 5 to calculate the horizontal difference between the solid curves and the dashed ones (along the $x$-axis) as a function of the hole depth (along the $y$-axis). The result is presented in Fig. 6 for $\theta=60^{\circ}$. We clearly see that for a certain erosion time, the additional erosion due to rebounding particles is almost constant, moreover, secondary particle underetching increases about linearly with etching time for a certain incidence angle, as is clear from Fig. 7. The points of Fig. 7 represent the experimental data, while the lines are just guides to the eye.

\subsection{Interpretation}

It is interesting to directly compare the secondary particle erosion contribution, shown in Fig. 7, with the hole depth. Dividing the values of Fig. 7 by the related hole depths, generates the curves of Fig. 8(a) for angles of incidence varying from 40 to $80^{\circ}$. The points are experimental data, while the lines represent the mean values for a given angle of incidence. The fact that the relative secondary particle erosion is independent of etching time and constant for a given angle of incidence, allows to design and exploit controlled underetching processes for future three-dimen-

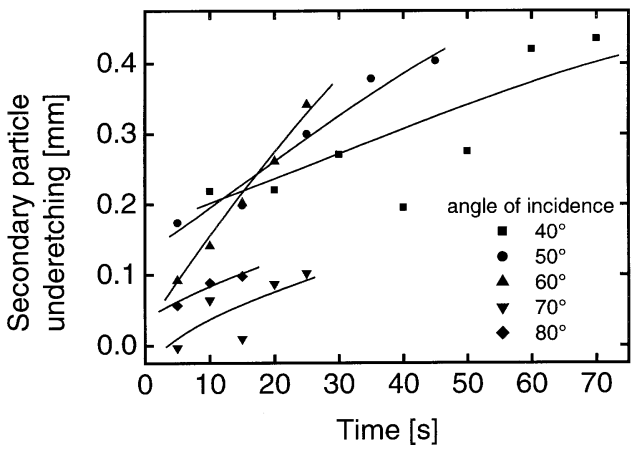

Fig. 7. Secondary particle underetching at the mask-substrate interface as a function of the etching time and for different angles $\theta$.
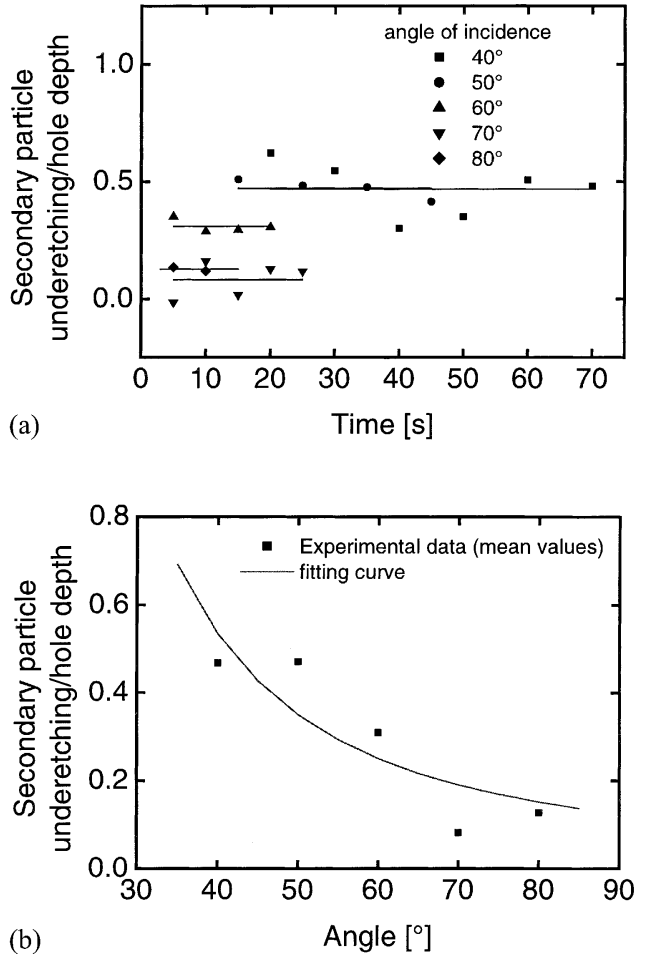

Fig. 8. (a) Ratio of the secondary particle underetching to the hole depth as a function of the etching time with the angle of incidence $\theta$ as parameter. (b) Evolution of the mean values of Fig. 8(a) with the angle $\theta$. The line is a fitting curve obtained using Eq. (4).

sional microfabrication. For example, structures in glass with an aspect ratio of 10 as well as totally suspended monolithic grid structures were realised by the use and control of the underetching phenomenon. Such structures were realised by fixing the substrate on a turntable, which gives rise to a uniform erosion and underetching rate from all sides of the substrate.

We note from Fig. 8(a) that the relative secondary particle erosion increases with increasing $\theta$. This rather obvious result indicates the increasing transfer of momentum of the particles from the vertical to the horizontal direction. For a given particle velocity $v$, the contribution to the vertical erosion process along the $y$-axis can be written as

$v_{y}=v \sin (\theta)$

In the general case, the particles loose a part of their kinetic energy between the primary impact on the bottom of the hole structure and the secondary impact at the sidewall, resulting in a smaller rebound angle and smaller particle velocity $v^{\prime}$ [2]. As determined experimentally [2], the exit angle of a rebounding particle is about $\theta / 2$ for our values of $\theta$ $\left(40^{\circ} \leq \theta \leq 80^{\circ}\right)$. Therefore, the determining particle impact velocity for the erosion of the side wall of the hole (along the $x$-axis) is

$v_{x}=v^{\prime} \cos \left(\frac{\theta}{2}\right)$ 
By combining Eqs. (1) and (2), we find that

$\frac{v_{x}}{v_{y}}=\frac{v^{\prime}}{v} \frac{\cos (\theta / 2)}{\sin (\theta)}=\frac{v^{\prime}}{v} \frac{1}{2 \sin (\theta / 2)}$

As the erosion rate of the substrate material is linear with the kinetic energy of the indenting particle [2], and therefore, quadratic in the particle velocity, we find from Eq. (3) that

$\left(\frac{v_{x}}{v_{y}}\right)^{2}=\left(\frac{v^{\prime}}{v}\right)^{2} \frac{1}{4 \sin ^{2}(\theta / 2)}$

Fig. 8(b) presents the mean experimental values of Fig. 8(a) as a function of the angle of incidence, together with a fitting curve, according to Eq. (4). We find that $\left(v^{\prime} / v\right)^{2}=0.25$ for the prefactor, which indicates significant inelastic loss of momentum between an impact at the bottom of the hole and at the sidewall. One observes a good agreement between the angle dependence of the experimental values of Fig. 8(b) and the fitting curve, suggesting that this very simple and crude model for sidewall particle impact is, at least to first order, correct.

\section{Conclusion}

We have shown that the introduction of an oblique angle of incidence to the particle beam during powder blasting with a masked substrate gives rise to specific erosion side wall effects. We have investigated in detail the basics of the oblique powder blasting phenomenon and have quantified the secondary particle erosion contribution using a mathematical procedure. By this method, we have highlighted the major role of second particle impacts in the mask underetching effect. The fundamental understanding of underetching phenomena will permit to control the exact shape of micropatterned structures, making powder blasting a real interesting alternative for the realisation of three-dimensional structures in brittle materials.

\section{Acknowledgements}

We would like to thank Philips Research for financial and technical support for this research and especially P.J. Slikkerveer, P. Bouten and F. de Haas for useful discussions.

\section{References}

[1] P.J. Slikkerveer, P.C.P. Bouten, F.H. in't Veld, H. Scholten, Erosion and damage by sharp particles, Wear 217 (1998) 237-250.

[2] P.J. Slikkerveer, Mechanical etching of glass by powder blasting, $\mathrm{PhD}$ Thesis, Eindhoven University of Technology, 1999.
[3] E. Belloy, S. Thurre, E. Walckiers, A. Sayah, M.A.M. Gijs, Powder blasting as a new technology for inertial sensor fabrication, in: Proceedings of the Eurosensors XIII, The Hague, The Netherlands, 12-15 September 1999.

[4] E. Belloy, S. Thurre, E. Walckiers, A. Sayah, M.A.M. Gijs, The introduction of powder blasting for sensors and microsystem applications, Sens. Actuators A 84 (2000) 330-337.

[5] H. Wensink, J.W. Berenschot, H.V. Jansen, M.C. Elwenspoek, High resolution powder blast micromachining, in: Proceedings of the 13th International Conference on Micro Electro Mechanical Systems (MEMS-2000), Miyazaki, Japan, 23-27 January 2000, pp. 769-774.

[6] E. Belloy, A. Sayah, M.A.M. Gijs, Powder blasting for threedimensional microstructuring of glass, Sens. Actuators A 86 (2000) 231-237.

\section{Biographies}

E. Belloy was born in Geneva, Switzerland, in 1972. He received his diploma in microengineering in 1997 from the Swiss Federal Institute of Technology of Lausanne (EPFL). His diploma work was the study, realisation and characterisation of microtips on $\mathrm{Si}$ and glass substrates, in use for the detection of the electrical activity of nerves cells. Since April 1997, he works as research assistant in the Institute of Microsystems at EPFL. He has first developed the technology process for a new magnetic sensor based on the Hall effect. Then, he has developed a new technology based on flex-foil patterning and assembling, in order to realise magnetic devices. His actual research area is on study of micro-erosion by powder blasting.

A. Sayah received his degree in physics in 1988 from the University Mohammed V, Rabat, specialising in solid state physics. He obtained his AESA and DEA in microelectronics specialising in the technology of components in 1990 and 1992, respectively. From 1993 until 1996, he worked as a research assistant in the CNET-Bagneux (Paris) Laboratory where he received a $\mathrm{PhD}$ degree for the thesis entitled "Realisation of Silicon-based dielectrics optical waveguide on InP by photochemical deposition". In 1996, he joined the Applied Optics Institute (IOA) of the Swiss Federal Institute of Technology in Lausanne (EPFL) as a postdoctoral researcher. His research interests included the study and characterisation of optical fiber tips for scanning near field optical microscopy by liquid-phase etching. He participated in the characterisation of Bragg gratings produced in optical fibers by UV laser illumination. He is now with the Institute for Microsystems (IMS) at EPFL. His current research interests are in inertial sensors (gyroscopes), bio-sensors, and fabrication technologies.

M.A.M. Gijs received his degree in physics in 1981 from the Katholieke Universiteit Leuven, Belgium and his $\mathrm{PhD}$ degree in physics at the same university in 1986. He joined the Philips Research Laboratories in Eindhoven, The Netherlands, in 1987. Subsequently, he has worked there on micro- and nano-fabrication processes of high critical temperature superconducting Josephson and tunnel junctions, the microfabrication of microstructures in magnetic multilayers showing the giant magnetoresistance effect, the design and realisation of miniaturised motors for hard disk applications and the design and realisation of planar transformers for miniaturised power applications. He joined the Swiss Federal Institute of Technology Lausanne (Ecole Polytechnique Fédérale de Lausanne) in 1997 as a professor in the Institute of Microsystems of the Microengineering Department, where he is responsible for the Microsystems Technology group. His main interests are in developing technologies for novel inductive-type devices, new microfabrication technologies for microsystems fabrication in general and the development and use of microsystems technologies for biomedical applications in particular. 